\title{
Electrostatic flat-top solitons near double layers and triple root structures in multispecies plasmas: How realistic are they?
}

Cite as: Phys. Plasmas 27, 062306 (2020); https://doi.org/10.1063/5.0001531

Submitted: 19 January 2020. Accepted: 02 June 2020 . Published Online: 18 June 2020

Frank Verheest (D), Manfred A. Hellberg (D), and Carel P. Olivier (D)
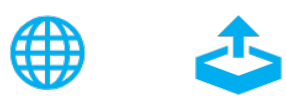

View Online

Export Citation

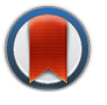

CrossMark

\section{ARTICLES YOU MAY BE INTERESTED IN}

Overtaking collisions of double layers and solitons: Tripolar structures and dynamical polarity switches

Physics of Plasmas 27, 062303 (2020); https://doi.org/10.1063/5.0003493

Diversity of solitary electron holes operating with non-perturbative trapping

Physics of Plasmas 27, 062302 (2020); https://doi.org/10.1063/5.0007941

Electron-exchange potential correction on dynamics of multidimensional ion acoustic waves in quantum plasmas

Physics of Plasmas 27, 062305 (2020); https://doi.org/10.1063/1.5144761

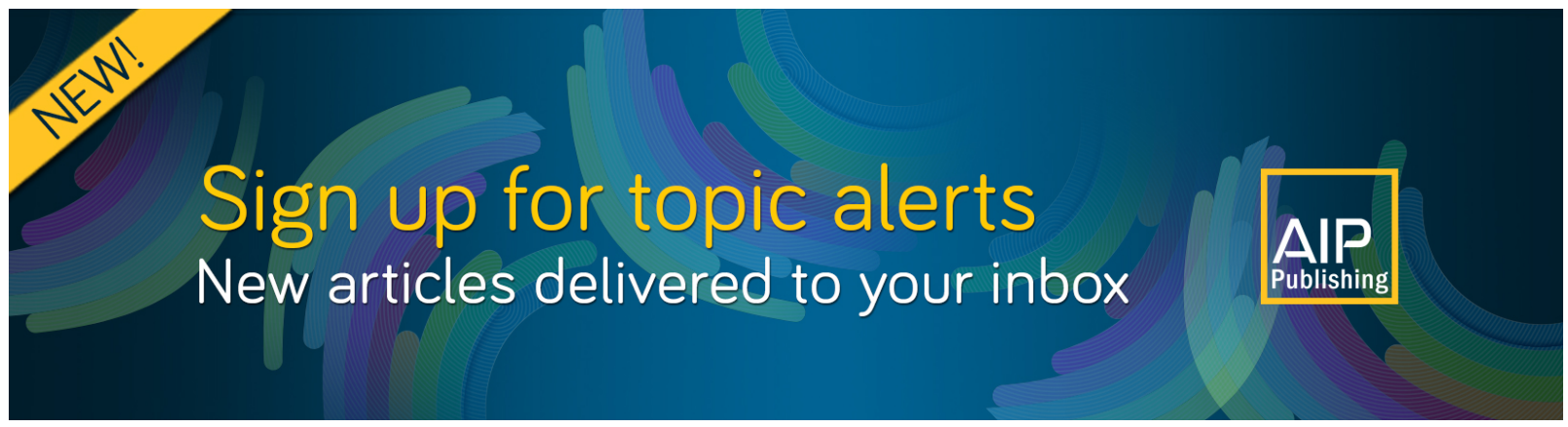




\title{
Electrostatic flat-top solitons near double layers and triple root structures in multispecies plasmas: How realistic are they?
}

Cite as: Phys. Plasmas 27, 062306 (2020); doi: 10.1063/5.0001531

Submitted: 19 January 2020 - Accepted: 2 June 2020 •

Published Online: 18 June 2020

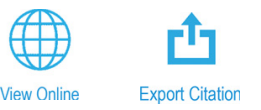

Frank Verheest, ${ }^{1,2, a)}$ (D) Manfred A. Hellberg, ${ }^{2, b)}$ (D) and Carel P. Olivier ${ }^{3, c)}$ (D)

\author{
AFFILIATIONS \\ ${ }^{7}$ Sterrenkundig Observatorium, Universiteit Gent, Krijgslaan 281, B-9000 Gent, Belgium \\ a) Author to whom correspondence should be addressed: frank.verheest@ugent.be \\ b)email: hellberg@ukzn.ac.za \\ c)email: Carel.Olivier@nwu.ac.za
}

${ }^{2}$ School of Chemistry and Physics, University of KwaZulu-Natal, Scottsville, Pietermaritzburg 3209, South Africa

${ }^{3}$ Department of Mathematics and Applied Mathematics, North-West University, Mafikeng Campus 2745, South Africa

\begin{abstract}
Electrostatic flat-top solitons are a new acoustic-type nonlinear mode and found to be a generic feature accompanying the occurrence of double layers and/or triple root structures, in multispecies plasmas admitting the latter. Their existence domains can be parameterized by the difference between their velocities and the double layer or triple root velocities, but these velocity differences turn out to be extremely small, of the order $10^{-5}$ or less. The onset of their flat top character in the electrostatic potential is clearly seen in the corresponding electric field or charge density profiles. However, even at the limit of the numerical accuracy for vanishing velocity differences, their profiles are still solitonlike, very unlike those of double layers or triple root structures. So although the Sagdeev potential varies continuously as the structure velocity approaches that of the double layer or triple root structure, the character of the nonlinear modes changes in a discontinuous manner. For sufficiently wide flat-top solitons, the electric field signature looks very much like two unipolar signals with opposite polarities, where unipolar electric fields typically characterize double layers or triple root structures. We are not aware of flat-top solitons having been reported to date, and their extremely limited existence range raises the question of whether they may be observable at all, unless helped by a fortunate stroke of serendipity. This topic requires suitable numerical simulations to ascertain their stability and interaction properties.
\end{abstract}

Published under license by AIP Publishing. https://doi.org/10.1063/5.0001531

\section{INTRODUCTION}

From a plethora of studies in the literature, it is well known that in several multispecies plasmas, a range of electrostatic acoustic-type solitary potential waves (sometimes simply called solitons for short, even when their interaction properties are not established) ends at a double layer of the same polarity. One of the commonly used tools is the Sagdeev pseudopotential analysis, ${ }^{1}$ which indicates that a range of solitons ends when the velocities and amplitudes of the solitons increase to those of the double layer. Whereas solitons correspond to single nonzero roots of the Sagdeev pseudopotential, a double layer is characterized by a double root outside equilibrium. This occurs when for a given set of plasma compositional parameters, such as densities, temperatures, masses, and charges, one considers an increase in soliton speed or an equivalent formulation in normalized units, a Mach number $M$. Given the vastness of the literature, we will only cite directly relevant papers.

While for a long time, a double layer was thought to be the end of a range of solitons, Dubinov and co-authors ${ }^{2,3}$ showed that in specific multispecies plasmas, a new type of soliton can exist beyond the double layer, called a supersoliton. That has a different character, with characteristic wiggles on its electric field profile. ${ }^{4-11}$ For such supersolitons, the Sagdeev potentials vary in a continuous way as the velocities increase from the double layer velocity, but the amplitudes show a distinct jump from the double layer amplitude to a larger amplitude range.

Conversely, the details of the transition from the soliton properties, for velocities smaller than the double layer velocity, to the double layer values has not been given much attention, until Steffy and 
Ghosh $^{12}$ investigated this range. Flat-top soliton potential profiles were shown to arise for velocities marginally smaller than the double layer velocity, with the degree of smallness being evaluated in terms of two small parameters, ${ }^{12}$ representing the first and second derivatives of the Sagdeev pseudopotential.

Exploring, thus, further the limits of the Sagdeev pseudopotential analysis, we give below, first, a clear discussion of several of the flat-top soliton properties and occurrence ranges, in terms of one small parameter with an obvious physical interpretation, namely, the difference between the double layer and the flat-top soliton velocities.

Second, we show in Appendix A that flat-top solitons are a necessary and generic accompaniment of double layers and triple root structures for different multispecies plasma models. Such a conclusion also holds when double layers are studied with the help of the Gardner equation in a reductive perturbation technique ${ }^{13}$ although the mathematical details are omitted from our paper itself. Reductive perturbation methods can only deal with weaker-amplitude solitons, and are thus of less importance for those plasma models which can be dealt with through a Sagdeev pseudopotential analysis.

Third, we show that although the Sagdeev potential varies continuously as the velocity difference between the flat-top solitons and the associated double layer goes to zero, the profiles show a discontinuous change in character from solitons (albeit flat-topped) to double layers.

To proceed, we use a general property of all plasmas in a Sagdeev-type energy integral description, ${ }^{1}$ for which the inertial species have barotropic pressure-density relations, ${ }^{14}$ namely, that an increase in $M$ means an increase in the (absolute) value of the first accessible root in potential, $\varphi$. This property, known from reductive perturbation theory that larger solitons are faster, is, thus, translated to larger-amplitude waves, even if for the latter one cannot obtain analytical expressions. In other words, without changing the boundary conditions at a stationary equilibrium, a continuous increase in $M$ means a continuous increase in soliton amplitudes (in absolute value), until this range ends. This can be because a physical restriction intervenes, or until a double layer is encountered. One recalls that the electrostatic potential profiles of both double root and triple root structures differ greatly from those of solitons. This also holds for the profiles of their associated electric fields $E$ and charge densities $\sigma$.

Formulated differently, for a Sagdeev pseudopotential with two successive roots of the same polarity (at $M<M_{d l}$ ), the one nearest to the origin is accessible from the undisturbed equilibrium at $\varphi=0$, representing a soliton, while the other, further away, is inaccessible and hence of no physical consequence. These two roots approach each other when $M$ is increased and ultimately coalesce at $M_{d l}$ as $M \rightarrow M_{d l}$. This shows up on the Sagdeev pseudopotential curve as a second double root, outside the trivial one at $\varphi=0$. As long as the double layer is not reached, for $M \rightarrow M_{d l}$ but such that $M<M_{d l}$, one finds solitons. However, for $M$ sufficiently close to $M_{d l}$, their profile in $\varphi$ changes to what has recently been called a flat-top solitary wave. ${ }^{12}$

This raises an interesting point: a flat-top solitary wave remains soliton-like, with profiles for $\varphi$ and $\sigma$ that are symmetric in $\xi$ around $\xi=0$, where $\xi$ is the "space" variable in a frame co-moving with the nonlinear wave, wherein it appears stationary. For the typical example, which we will discuss below, the flattop becomes noticeable when $M_{d l}-M<10^{-4}$ and holds even for $M_{d l}-M=10^{-16}$. Even then, the profiles are not those of a double layer although we are so close to the double layer conditions so as to reach the limits of numerical accuracy and reliability. So it appears that, although the Sagdeev pseudopotential changes continuously when $M \rightarrow M_{d l}$, the character of the profiles changes discontinuously at the double layer velocity itself.

Similar remarks can be made about solitons near triple root structures, in those plasmas where these can be encountered. ${ }^{15}$ Hence, when we use "near," it is meant in terms of $M$ in parameter space, not in a physical sense.

This paper is organized as follows. After this introduction, Sec. II deals with our analysis of a range of solitons near a double layer, in the region where the solitons exhibit a flat-top profile in $\varphi$. A similar exercise is then given in Sec. III for flat-top solitons near triple root structures. Given the extremely narrow range in $M$ to obtain flat-top solitons, around the exact limit for having double layers or triple root structures, the question arises as to the chances of observing or realizing such structures, given the unavoidable noise or limits to the numerical accuracy. This is discussed in Sec. IV. Finally, Sec. V briefly summarizes our conclusions. Appendix A contains the analytical proof that flat-top solitons are generic to double layers and triple root structures, while some of the mathematical details about the models used to illustrate our findings have been relegated to Appendixes B (double layers) and $C$ (triple root structures).

\section{FLAT-TOP SOLITARY WAVES NEAR DOUBLE LAYERS}

As shown in Appendix A, flat-top solitons are a generic occurrence before arriving at a double layer, and therefore, one can choose any of the multispecies plasma models that admit double layers to illustrate the different aspects of the reasoning. Thus, for example, for the sake of graphical illustration, we return to a model used earlier, ${ }^{16}$ for which we had carried out an exhaustive parametric investigation of the existence domains in compositional parameter space, to determine where and under what conditions double layers could be generated. ${ }^{16}$ Thus, an appropriate set of parameter values can be picked, without relying on the trial-and-error procedure that is used all too often.

For completeness, we recall here the basic elements for dustacoustic solitary structures, which have been used to generate the figures illustrating this section. This dusty plasma composition contains three species: hot electrons, Cairns nonthermal ${ }^{17}$ positive ions, and cold, negatively charged dust grains. For more details, we refer to the original paper. ${ }^{16}$ Some of the steps leading to the energy integral are briefly recalled in Appendix B, where the relevant compositional parameters are also introduced. The Sagdeev pseudopotential for this model is ${ }^{16}$

$$
\begin{aligned}
S(\varphi, M)= & \frac{1-f}{\tau}(1-\exp [\tau \varphi])+f M^{2}\left(1-\sqrt{1+\frac{2 \varphi}{M^{2}}}\right) \\
& +1+3 \beta-\left(1+3 \beta+3 \beta \varphi+\beta \varphi^{2}\right) \exp [-\varphi] .
\end{aligned}
$$

All Sagdeev pseudopotentials for electrostatic modes contain a number of plasma compositional parameters. Through a judicious normalization of the variables, these may often be reduced to a number of dimensionless ratios, as for densities, temperatures, or masses. These determine the (multispecies) plasma model. Besides those plasma parameters, there are two other variables: the electrostatic potential $\varphi$ and the soliton velocity, normalized as $M$. These are not known $a$ priori and must be determined according to whether one wants to obtain a soliton or a double layer. For double layers, one looks for nontrivial sets $\{\varphi, M\}$, which satisfy both equations 


$$
S(\varphi, M)=0, \quad S^{\prime}(\varphi, M)=0,
$$

where the compositional plasma parameters have not explicitly been mentioned and the prime denotes a derivative of $S(\varphi, M)$ with respect to $\varphi$. In principle, this works very well and the numerical accuracy is high.

The property that $\partial S(\varphi, M) / \partial M<0$, for any given plasma composition in which the inertial species have barotropic pressures, ${ }^{14}$ means that the Sagdeev pseudopotentials form a one-parameter family of curves, outside the equilibrium at $\varphi=0$. Thus, the pseudopotential curves cannot cross, and an increase in $M$ lowers the curve, at any given $\varphi$. This influences the roots, a property that will come in handy when discussing the surroundings of double layers and triple roots.

Suppose that there is a double root (outside the equilibrium), as seen, e.g., in Fig. 1, for $M=M_{d l}$, which implies that $S(\varphi, M)<0$ on both sides for $\varphi \neq \varphi_{d l}$ in a sufficiently small neighborhood, where $\varphi_{d l}$ is the double root. An increase in $M$ above $M_{d l}$ lowers the curve, and the Sagdeev pseudopotential will have two fewer roots above $M_{d l}$. As mentioned already in the Introduction, for specific plasma compositions, there might be an accessible root further away from $\varphi=0$, but such a supersoliton has a different character. ${ }^{2-11}$ Supersoliton roots are, in general, too far away from the double layer to be able to lead to flat-top profiles. For supersolitons beyond a double layer, there is a discontinuous jump in amplitude when $M$ increases smoothly above $M_{d l}$. Thus, flat-top solitons are not found for $M>M_{d l}$.

On the other hand, for reasons of continuity, decreasing $M$ below $M_{d l}$ results in two roots, one accessible from the undisturbed equilibrium, smaller than $\varphi_{d l}$ and representing a solitary wave, and the other one larger than $\varphi_{d l}$ and, thus, inaccessible from $\varphi=0$ and of no physical relevance. Figure 1 illustrates this graphically.

What will happen, as seen below, is that for $M<M_{d l}$ but close enough to $M_{d l}$, the solitons develop a flattop. This immediately excludes flat-top solitons for plasma compositions where a double layer occurs at the acoustic speed $M_{s},{ }^{10}$ as any $M<M_{s}=M_{d l}$ gives no solutions. The flat top part of the soliton potential profile becomes larger as one gets close to the double layer, but what happens precisely in the limit $M \rightarrow M_{d l}$ is beyond the numerical precision of the plotting routines. It has to be remembered that, in general, an analytical form or function for the soliton profile is not available.

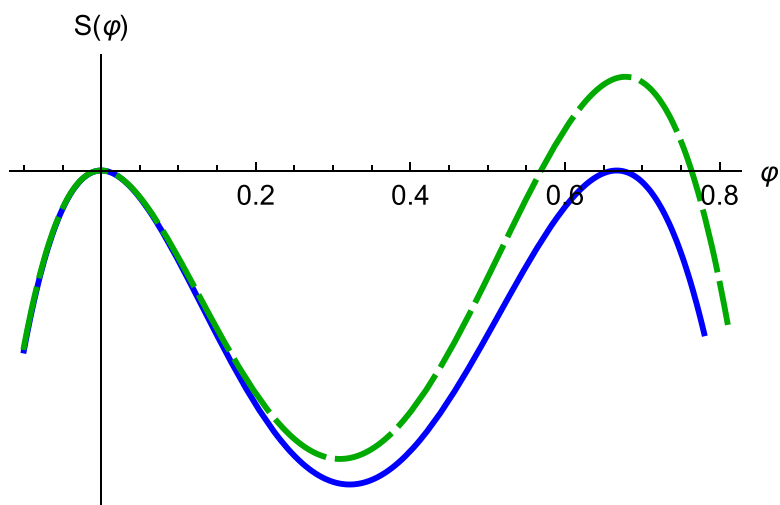

FIG. 1. Sagdeev pseudopotentials having double layer (blue curve) and standard solitary wave (dashed green curve) solutions.
Moreover, the change from normal to flat-top soliton profiles when close enough to a double layer (and below, to a triple root structure) is generic, valid for all multispecies plasmas that can be investigated through a Sagdeev pseudopotential analysis, as proved in Appendix A. Thus, they are not an artifact for a specific model. For completeness, we have checked this for models in our own papers for which we readily had the numerics. ${ }^{6,7,14,18-22}$ It transpired that flat-top solitons are clearly noticeable near double layers, for around $M_{d l}-$ $M=10^{-6}$ and smaller. The graphs illustrating this are not included in this paper for lack of space and because they look repetitious. The models in question include Boltzmann, ${ }^{18,21,22}$ Cairns,,$^{14,19-22}$ and kappa $^{7}$ electrons and Boltzmann ${ }^{18,21}$ and Cairns ${ }^{14,19,21}$ protons, for the inertialess hot species. The cooler species with inertia are cold ion spe$\operatorname{cies}^{6,7,14,19,20,22}$ and $\operatorname{cool}^{6,18,21}$ and adiabatic ${ }^{18}$ negative dust. In addition, of course, there is the model used by Steffy and Ghosh, having two Boltzmann electron species and two adiabatic positive ion species. $^{12}$

A similar test has also been done for triple root structures, ${ }^{21}$ the properties of which are discussed in Sec. III. Hence, the graphical illustrations presented in this paper, based on specific plasma models, are nevertheless generic and qualitatively representative for multispecies plasmas admitting double layers (for Sec. II) or triple root structures (for Sec. III). Precisely because the focus is on general, qualitative aspects, we omit the numerical details of the plotting routines, as these play no crucial role in our arguments.

To begin with, we present in Fig. 2 typical curves illustrating (a) the potential profile of a double layer, (b) its associated electric field, and (c) charge density. We note that the electric fields associated with a double layer typically have unipolar signatures, as opposed to the bipolar signatures of solitons. The black dotted curves in Fig. 3 show the characteristic soliton potential and electric field profiles. Observers tend to record electric fields and, hence, define the appearance of solitons and double layers in terms of those. However, in the standard Sagdeev description, one tends to look at potentials and the potential profiles, so that our figures show first of all $\varphi$. This will also influence the discussion of the structure polarities, based here on $\varphi$.

To try and understand what happens to solitary electrostatic waves when $M \rightarrow M_{d l}$, we illustrate in Fig. 3 some intermediate profiles, for increasing soliton velocities, smaller than, but getting closer to the double layer velocity. The intermediate profiles given in Fig. 3 are for (a) solitary wave potentials, (b) associated electric fields, and (c) charge densities, for soliton velocities approaching the double layer velocity from below. In each figure, the dotted black curves correspond to $M_{d l}-M=10^{-3}$, the dashed green to $M_{d l}-M=10^{-6}$, and the full red to $M_{d l}-M=10^{-16}$, all for $M<M_{d l}$.

It is immediately seen that the soliton potential profile broadens near the top and that the electric field shows inflections near the origin in $\xi$ that grow more pronounced as the velocity increases toward that of the double layer.

Interestingly, the dotted black curves for $M_{d l}-M=10^{-3}$ for both the potential and the electric field are reminiscent of those of a standard soliton, not of a flat-top soliton. However, the corresponding charge density indicates that it is no longer a simple soliton, given the indentation near the top at $\xi=0$. We note that for a standard soliton, the charge density has a definite tripolar shape, with a peak at the origin.

At $M_{d l}-M=10^{-6}$, the profile of a flat-top soliton in $\varphi$ emerges, there is marked inflection of the $E$-field at the origin, and 

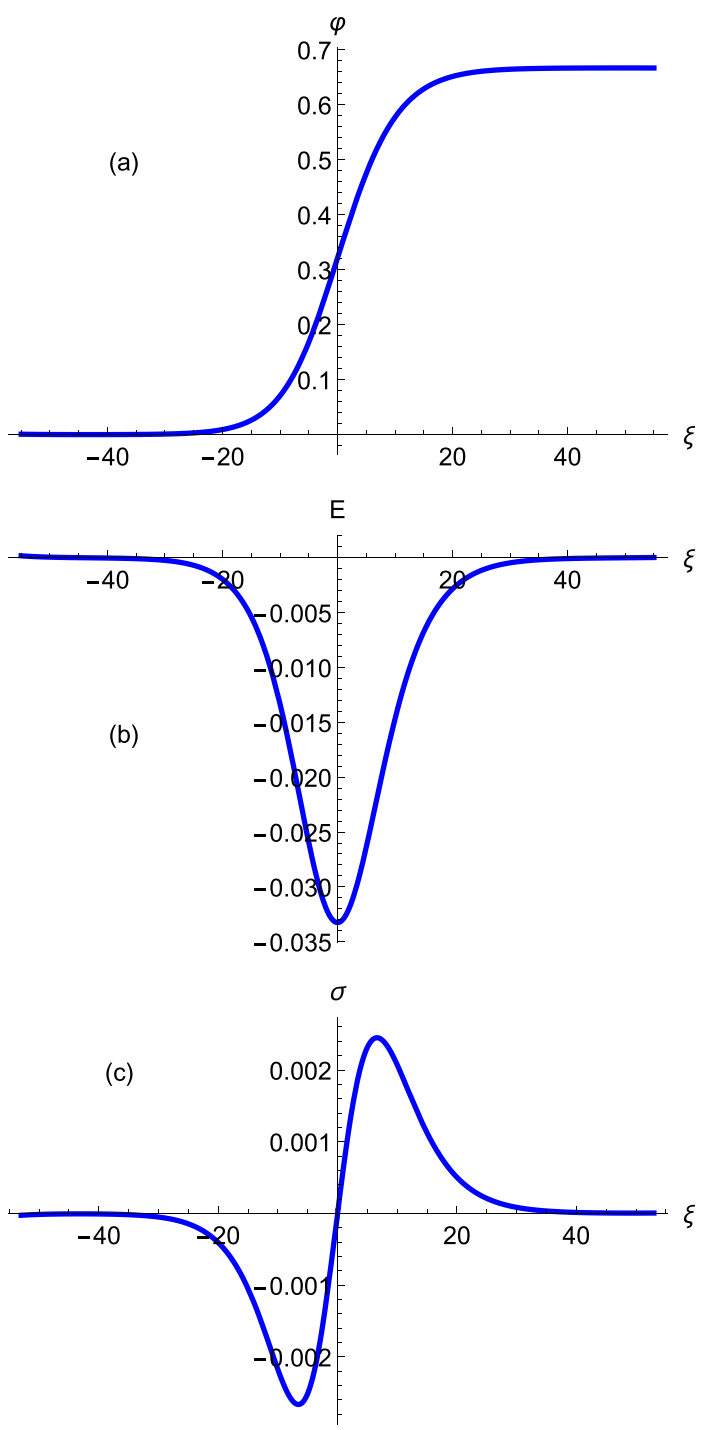

FIG. 2. Typical profiles for (a) double layer potential structure, (b) its electric field, and (c) charge density.

the dip in what would be the peak in the charge density for a standard soliton goes down to zero. This behavior grows more and more pronounced as $M$ approaches $M_{d l}$ more closely, as shown by the red curves for $M_{d l}-M=10^{-16}$. But, even in such close proximity to the conditions supporting a double layer, the curves are significantly different from those of Fig. 2.

As $M_{d l}-M$ decreases, it is seen on the electric field profiles that the bipolar character tends to morph into two quasi-unipolar profiles, separated by the width of the flattop in the potential profile, in which region both the electric field and the charge density vanish. The two quasi-unipolar profiles characteristically have opposite polarities, however, in contrast to that of the double layer at $M_{d l}$.

If we plot the corresponding Sagdeev pseudopotentials on the usual scale, as in Fig. 4(a), only the dotted black curve is distinct from the blue curve for a double layer. The dashed green and full red curves
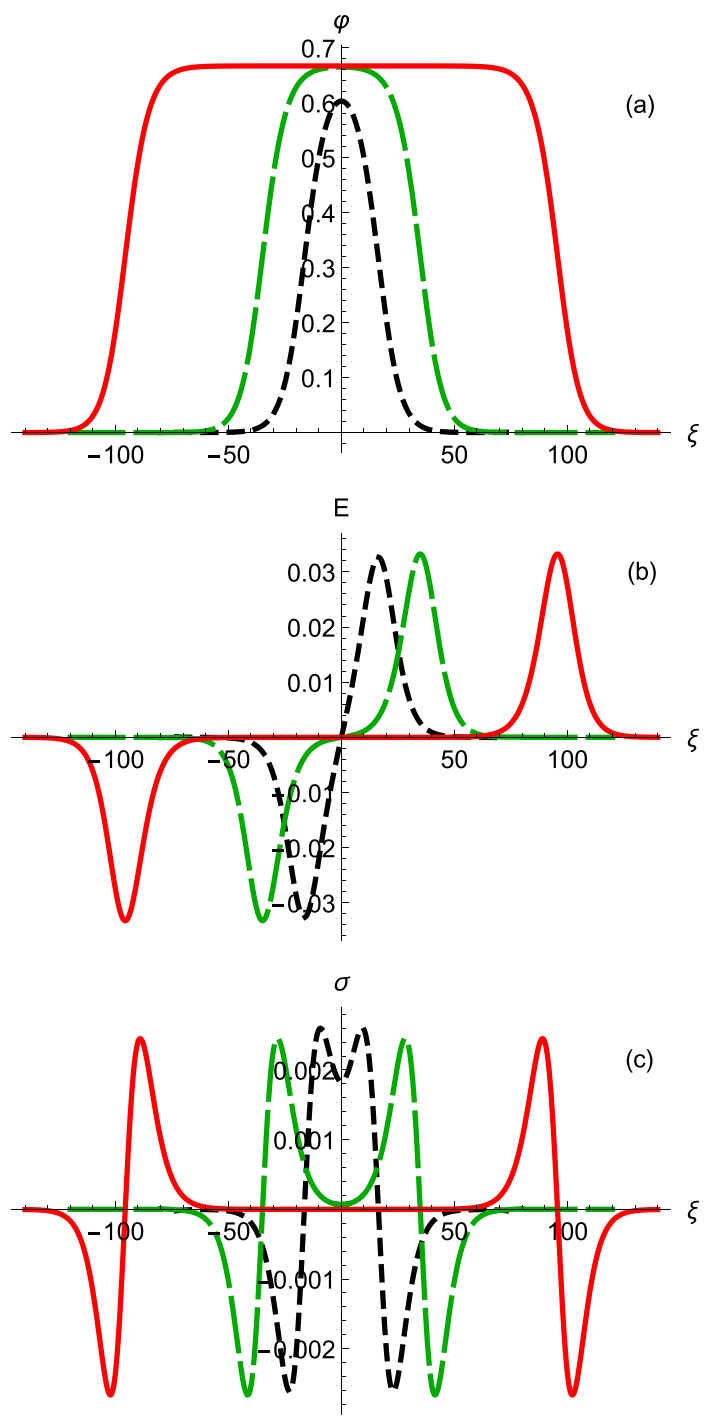

FIG. 3. Intermediate profiles of (a) solitary wave potentials, (b) electric fields, and (c) charge densities, for soliton velocities approaching the double layer velocity from below. The dotted black curves correspond to $M_{d l}-M=10^{-3}$, the dashed green to $M_{d l}-M=10^{-6}$, and the full red to $M_{d l}-M=10^{-16}$, all for $M<M_{d l}$.

are indistinguishable from the double layer curve. In Fig. 4(b), we restrict ourselves to only a small range in $\varphi$ around $\varphi_{d l}$, and the dotted black curve has been omitted for graphical clarity, as being too large on the scale used to visualize the small changes. Now, the dashed green curve $\left(M_{d l}-M=10^{-6}\right)$ is distinct from that of the double layer, but the red curve is still too close to the blue one to be distinguishable. Yet, even though the Sagdeev pseudopotentials are graphically indistinguishable at this level, with $M_{d l}-M=10^{-16}$, the soliton potential profile is still that of a symmetric, soliton-like flat-top structure, not that of a double layer, which is asymmetric, with different values at $\xi \rightarrow-\infty$ and $\xi \rightarrow+\infty$. Diminishing the difference in $M$ even more is at the limit of the numerical accuracy needed to produce the graphs, for which we, unfortunately, do not have analytical expressions. 
As an aside, we also tested the results for oblique propagation of electrostatic solitons with respect to an external magnetic field ${ }^{23}$ and, remarkably, found that there, too, flat-top solutions emerged near a double layer. However, the theoretical development of electrostatic nonlinear modes at oblique propagation in magnetized plasmas relies on assumptions about quasineutrality and the neglect of wave magnetic effects, which imply stringent restrictions on the acceptable ranges of amplitudes, ${ }^{24}$ the validity of which is seldom if ever checked a posteriori. Purely electromagnetic flat-top waves have also been discussed, ${ }^{25}$ but these are clearly outside the focus of the present paper.

Our results on flat-top solitary waves correspond qualitatively to those presented by Steffy and Ghosh. ${ }^{12}$ In their paper, the degree of how close one is in parameter space to the double layer has been expressed in terms of (in our notation) two small parameters $\varepsilon=\partial S /\left.\partial \varphi\right|_{\varphi_{\text {d }}}$ and $\delta=\partial^{2} S /\left.\partial \varphi^{2}\right|_{\varphi_{\text {I }}}$. We have preferred to use one smallness parameter with a clear physical interpretation, namely, the absolute value of the velocity difference between the double layer (or, below, the triple root structure) and its neighboring flat-top solitons. As discussed, this velocity difference is tiny.

A final note: we have assumed that the nonlinear structures start as infinitesimal perturbations of the unstable equilibrium at $\varphi=0$ and $\partial \varphi / \partial \xi=0$. For solitons, the boundary conditions on the electrostatic potentials are the same left and right, going as $\varphi \rightarrow 0$ for $\xi \rightarrow \mp \infty$. The range of solitons occurring for increasing $M$ ultimately ends when a double layer is encountered at $M_{d l}$. For a double layer, the boundary conditions left and right are different, in our case [Fig. 2(a)], $\varphi \rightarrow 0$ for $\xi \rightarrow-\infty$ and $\varphi \rightarrow \varphi_{d l}$ for $\xi \rightarrow+\infty$.

As our study shows, the transition from (flattop) solitons to double layers (or, for that matter, to triple root structures discussed in
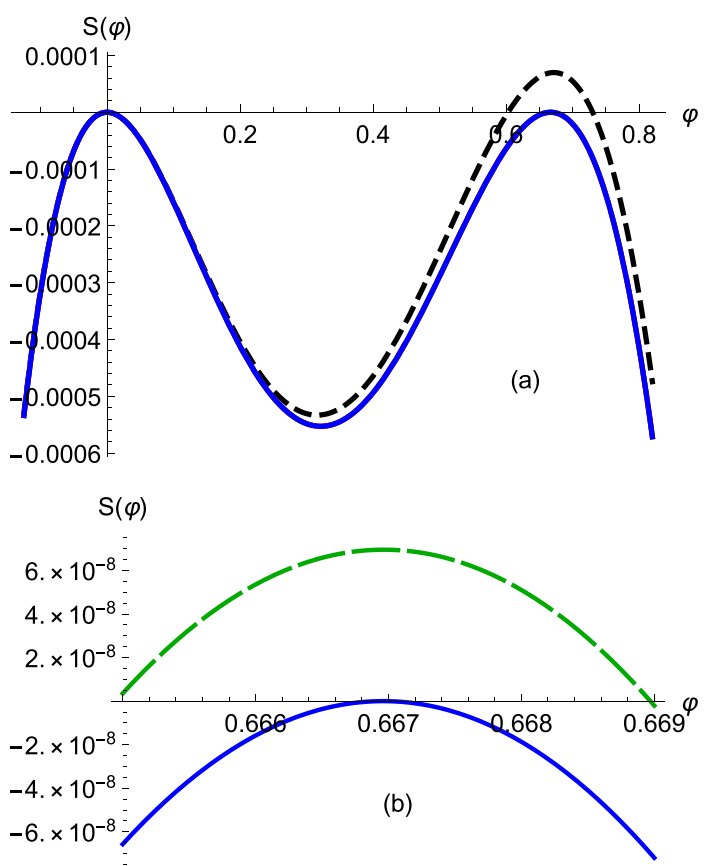

FIG. 4. Sagdeev pseudopotentials for which the double layer profiles are shown in Fig. 2. Part (a) is general and (b) an enlargement of the $\varphi$ region around the double layer root. The colors correspond to the profiles shown in Figs. 2 and 3.
Sec. III) is discontinuous in character even though for a given plasma composition, the Sagdeev pseudopotential varies continuously with $M$. The reason for this is that the flat-top profiles, even for wide flattops, clearly show soliton boundary conditions, as far as our numerical precision allows. Thus, it is difficult to sustain the claim that flat-top solitons are amalgamations of a solitary wave and a double layer ${ }^{12}$ or that a very wide flat-top soliton can be viewed as two double layers of different polarity propagating back to back.

\section{FLAT-TOP SOLITARY WAVES NEAR TRIPLE ROOT STRUCTURES}

First of all, we recall that in certain configurations, the Sagdeev pseudopotential can have triple roots, which have similar profiles to those of double layers, ${ }^{15}$ except that the approach to the triple root value is algebraic rather than exponential as it is for double layers. ${ }^{26}$ Another distinction from double layers is that triple root structures occur inside a range of solitons, rather than signaling the end of such a range.

We shall consider a dusty plasma model that, in principle, includes Cairns nonthermal electrons and ions, and cold negative and positive dust grains. ${ }^{15}$ However, we seek to use a model that is as simple as possible to illustrate the present paper, rather than complicating the presentation with details that are not essential. Thus, we shall take the electrons and ions as Boltzmann rather than nonthermal Cairns distributed. Between parentheses, this special case has also been worked out in the original paper. ${ }^{15}$ The corresponding Sagdeev pseudopotential is, thus,

$$
\begin{aligned}
S(\varphi, M)= & f M^{2}\left(1-\sqrt{1+\frac{2 \varphi}{M^{2}}}\right)+(1-f)\{1-\exp [\varphi]\} \\
& +g \mu M^{2}\left(1-\sqrt{1-\frac{2 \varphi}{\mu M^{2}}}\right)+(1-g)\{1-\exp [-\varphi]\} .
\end{aligned}
$$

In Appendix $\mathrm{C}$, we outline the steps taken to reach this function and also introduce the notation used.

To find triple roots is more intricate than for double layers because three equations have to be used in order to find a common set of values,

$$
S(\varphi, M)=0, \quad S^{\prime}(\varphi, M)=0, \quad S^{\prime \prime}(\varphi, M)=0 .
$$

In contrast to double roots, where, in principle, the compositional parameters might be freely chosen (within existence ranges) when determining the set of $\left\{\varphi_{d l}, M_{d l}\right\}$ from (2), obtaining triple roots requires one of the plasma compositional parameters to be fixed through (4), together with $\left\{\varphi_{t r}, M_{t r}\right\}$. As a consequence, determining whether a certain plasma can sustain a triple root structure is a more delicate exercise than finding double roots. Then, for flat-top solitons in the neighborhood of the triple root structure, one keeps the compositional parameter in question at this specific value, so that the plasma composition does not change when $M$ is near $M_{t r}$. One then, determines numerically the profiles in $\xi$, for speeds $M$ close enough to $M_{t r}$.

We now illustrate in Fig. 5 in (a) the profile of a triple root potential structure, in (b) its electric field, and in (c) the charge density. It is worth comparing this triple root structure with the double layer presented in Fig. 2(a). The left hand part of the graphs (negative $\xi$ ) is 

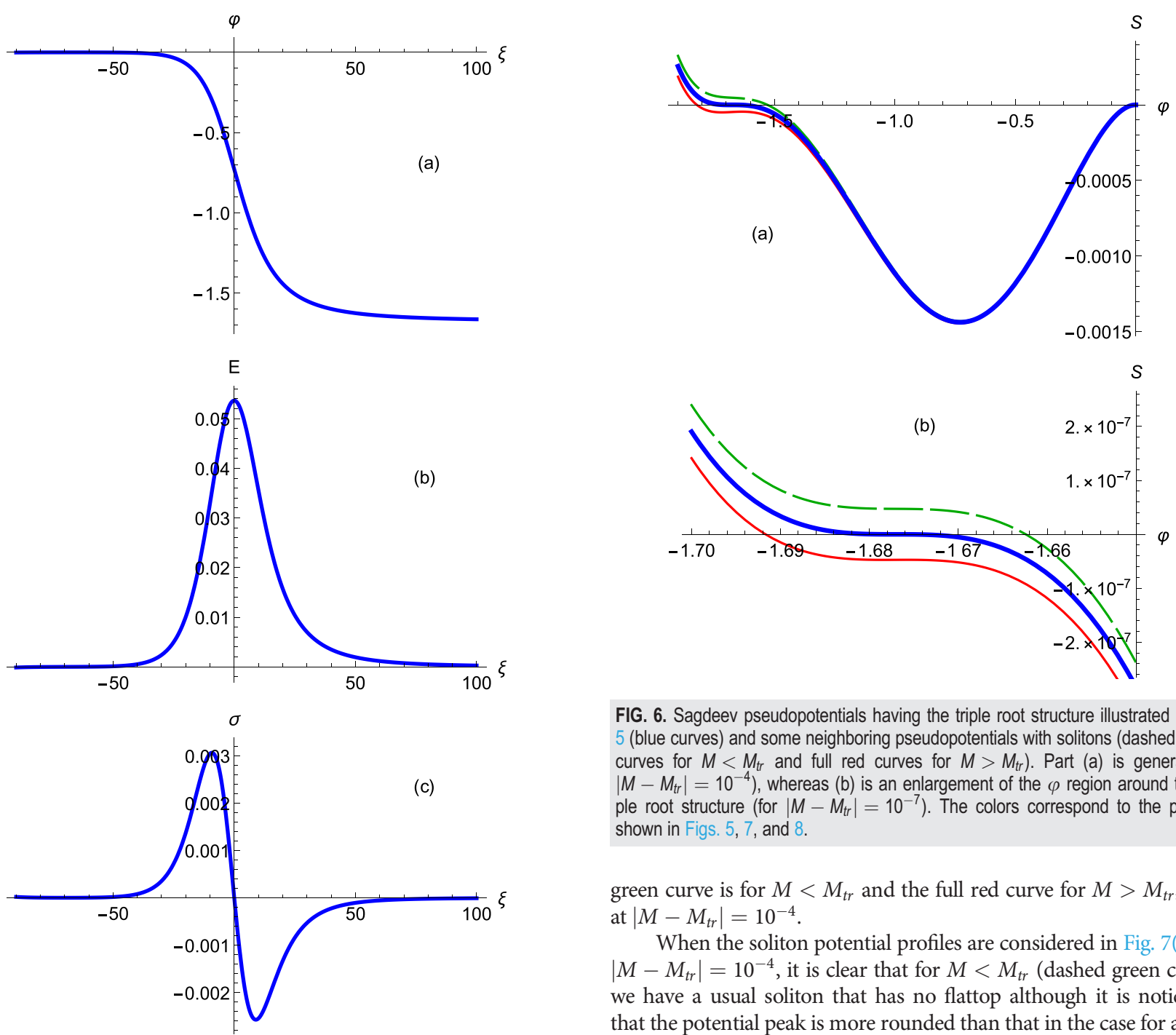

FIG. 5. Profiles of (a) triple root potential structure, (b) associated electric field, and (c) charge density.

similar, but for a change in polarity, as both emerge from the double root at $\varphi=0$. On the right-hand side, the double layer profile in Fig. 2(a) decays exponentially to another double root, whereas the triple root solution in Fig. 5(a) forces an algebraic decay. ${ }^{26}$ Again, the electric field profile presents a unipolar character. Although at first sight Figs. 2 and 5 look very similar, closer examination reveals that they do differ, most visibly in the slow rate of decay of the triple root profiles in the limit $\xi \rightarrow+\infty$.

Having ascertained that triple root structures exist at a speed $M_{t r}$ we try to understand what the neighboring solitons look like, for $M$ close to but different from $M_{t r}$. Importantly, we shall see that, contrary to what occurs near double layers, one can now have flat-top solitons in both the ranges $M<M_{t r}$ and $M>M_{t r}$.

We give an example of relevant Sagdeev pseudopotentials in Fig. 6(a), where the blue curve represents $M=M_{t r}$, while the dashed

FIG. 6. Sagdeev pseudopotentials having the triple root structure illustrated in Fig. 5 (blue curves) and some neighboring pseudopotentials with solitons (dashed green curves for $M<M_{\text {tr }}$ and full red curves for $M>M_{\text {tr }}$ ). Part (a) is general (for $\left.\left|M-M_{t r}\right|=10^{-4}\right)$, whereas (b) is an enlargement of the $\varphi$ region around the triple root structure (for $\left|M-M_{t r}\right|=10^{-7}$ ). The colors correspond to the profiles shown in Figs. 5, 7, and 8.

green curve is for $M<M_{t r}$ and the full red curve for $M>M_{t r}$, both at $\left|M-M_{t r}\right|=10^{-4}$.

When the soliton potential profiles are considered in Fig. 7(a) for $\left|M-M_{t r}\right|=10^{-4}$, it is clear that for $M<M_{t r}$ (dashed green curve), we have a usual soliton that has no flattop although it is noticeable that the potential peak is more rounded than that in the case for a typical standard soliton. The full red curve for $M>M_{t r}$, too, does not

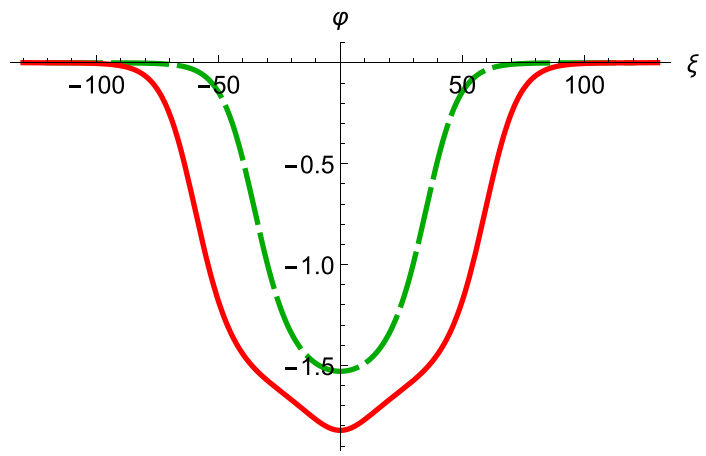

FIG. 7. Potential profiles of typical ordinary and supersolitary waves corresponding to the Sagdeev pseudopotentials shown in Fig. 6(a), for $\left|M-M_{t r}\right|=10^{-4}$, for which flat-top effects are not yet noticeable. 
have a flattop, but has a distorted soliton-like shape that is characteristic of a supersoliton. That is confirmed by noting the characteristic, albeit very shallow, subsidiary well within the usual Sagdeev potential well $^{5-8}$ as shown in Fig. 6(a). Their electric fields and charge densities are omitted here, in order not to overload the paper.

Turning now to Fig. 6(b), this enlargement is for smaller velocity differences, $\left|M-M_{t r}\right|=10^{-7}$. The potential profiles, electric fields, and charge densities are shown in Fig. 8 and clearly exhibit the flat-top character observed in Fig. 3. The differences between $M$ and $M_{t r}$ can be further reduced; until around $\left|M-M_{t r}\right|=10^{-9}$, we run into the limits of the numerical accuracy of our programming. The effect of lowering $\left|M-M_{t r}\right|$ is to broaden the flat-top part of the graphs, as observed above for structures related to double layers.
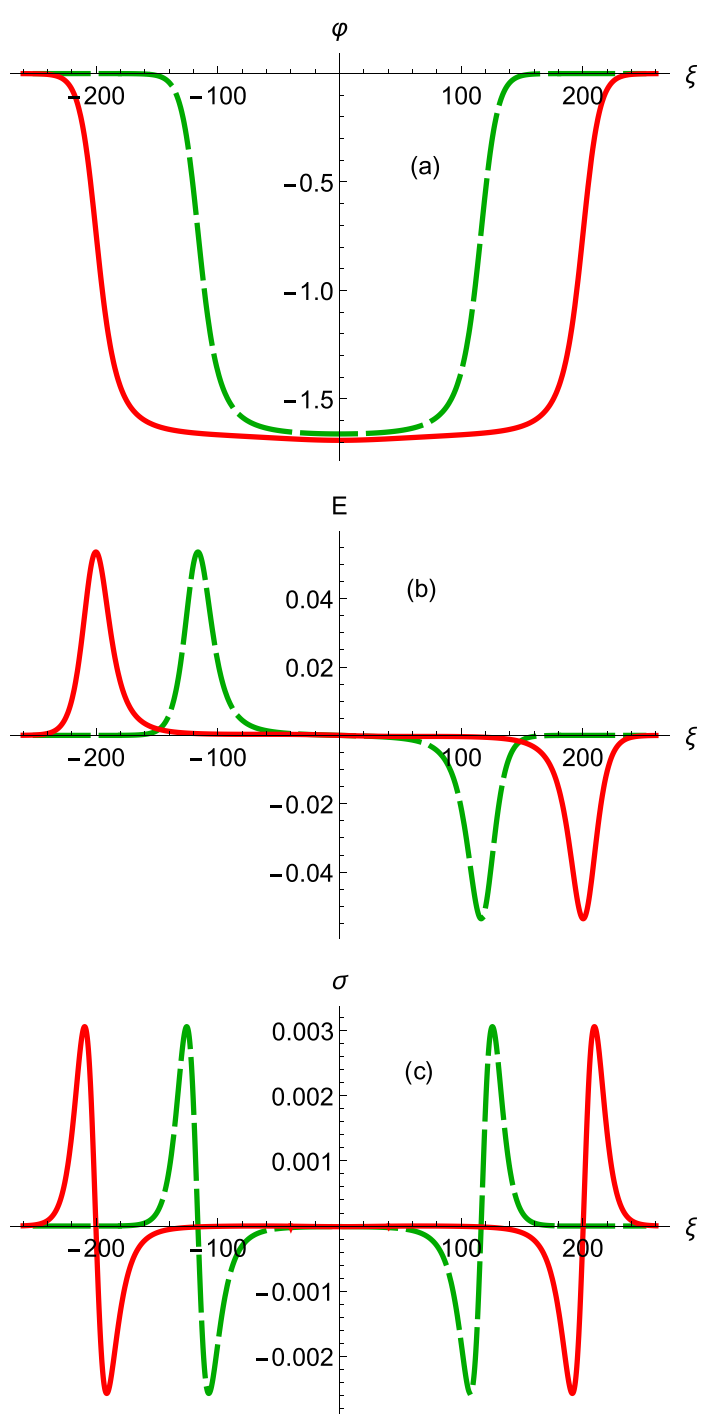

FIG. 8. Profiles of typical (a) solitary potential waves, (b) electric fields, and (c) charge densities, for flat-top solutions corresponding to the Sagdeev pseudopotentials shown in Fig. $6(\mathrm{~b})$, with the same color coding, but now for $\left|M-M_{t r}\right|=10^{-7}$ The triple root structure properties have been omitted for graphical clarity.
It, thus, looks as though, for $M>M_{t r}, M \rightarrow M_{t r}$, one has a sequence of supersolitons (for $10^{-4}$ ), via flat-top supersolitons (for $10^{-7}$ ) to a triple root. This is the reverse sequence from what happens for solitons for $M<M_{t r}, M \rightarrow M_{t r}$, and is equally generic.

\section{IMPLICATIONS FOR OBSERVABILITY AND PRESENCE}

For ranges of solitons which end at double layers, the soliton velocities may span quite an interval $M_{s}<M<M_{d l}$, whereas double layers (or triple root structures) require a very precise velocity, at least in the theoretical description used here for determining those. Clearly, double layers, triple root structures, and, thus, flat-top solitons also are very sensitive to small changes in $M$.

To relate our exposition to possible satellite observations in space plasmas, we recall that the power of the Sagdeev pseudopotential analysis is that it is fully nonlinear and can deal with large amplitudes. The drawback is that one can only study one mode at a time, without being able to investigate stability or interaction properties of that mode. On the other hand, reductive perturbation theory can treat nonlinear modes and include their stability and collisional properties, but is limited to amplitudes that are not too large, due to its iterative expansions and slow time scales.

Both methods have a common assumption that the nonlinear solitary mode has somehow been generated and has reached its final form. To go beyond these theoretical aspects of nonlinear modes, numerical simulations are needed, starting from the basic fluiddynamical equations or from kinetic descriptions, outside the scope of this paper. Such simulations have to be worked out for each specific plasma model composition at a time. They, thus, lack the general applicability of analytical methods such as reductive perturbation or Sagdeev pseudopotential theory, which can deal with a whole spectrum of multispecies plasma models.

Satellite observations in the auroral regions of the ionosphere have at first given many examples of bipolar electric field profiles or trains of well-separated bipolar signatures, of the same polarity. ${ }^{27,28}$ Similar observations sometimes mention weak double layers without significant net potential. ${ }^{29}$ Clear double layers have been observed, with their unmistakable unipolar electric field signatures, first in the auroral regions ${ }^{30,31}$ and later also in the plasma sheet of Earth's magnetosphere, ${ }^{32}$ to quote but some examples of the literature. Various mechanisms are invoked to generate such double layers and explanations involve numerical simulations.

One of the difficulties in trying to correlate theory and observations is that many space observations involve temporal measurements taken on board a single satellite as it moves through space. The resultant "snapshots" of nonlinear structures (soliton or double layer) provide no information about their propagation velocity. However, while the modeling and interpretation of the nonlinear profiles are based on the assumption of traveling wave modes, there are unfortunately only a small number of unambiguous observations by multi-spacecraft missions of actually propagating wave modes. ${ }^{33,34}$ The simple reason for this is that for positive identification, a passing wave structure has to be recorded by at least two spacecrafts, operating in the same instrumental mode and orientation.

So, double layers can exist even though the standard theoretical descriptions in terms of electrostatic modes seem to limit them to very particular combinations of $\varphi$ and $M$. Regarding the flat-top solitons, 
in se a very interesting theoretical development, we are not aware of any observations to date of structures that are identifiable as flat-top solitons, neither in space observations nor in laboratory studies. That might be understandable, as our numerical analysis has shown that they only exist in extremely narrow $M$ ranges, of the order of $M_{d l}-$ $M<10^{-5}$ or $\left|M-M_{t r}\right|<10^{-5}$. Moreover, even when going to lower limits on the deviations of $M$ from $M_{d l}$ or from $M_{t r}$ the flattops in the $\varphi$ profiles get broader and broader, but remain soliton-like (i.e., singlehumped, unlike the potential kinks or double layers), having the same boundary conditions left and right, until the numerical accuracy stops our computations.

It is possible that flat-top solitons might be very sensitive structures, as indicated by our analysis, and, thus, not be observable, while double layers might be inherently robust structures, more stable and, thus, observable. Hence, the stabilities of both double layers and flattop solitons are worth investigating using numerical simulation and stability analysis, both of which go beyond the scope of the present investigation.

\section{CONCLUSIONS}

Flat-top solitary waves were recently mentioned as a new nonlinear acoustic-type wave mode in certain multispecies plasmas. ${ }^{12}$ Their existence domains are intimately tied to the neighborhood of double layers or triple root structures, with the following properties:

1. We have chosen to parameterize flattops by the absolute value of the difference between their velocities and the double layer or triple root structure velocities, $M_{d l}-M$ or $\left|M-M_{t r}\right|$, having a simple physical interpretation.

2. From our investigations, it transpires that these flat-top solitons are a generic feature accompanying the occurrence of double layers and/or triple root structures, in multispecies plasmas admitting the latter. At the same time, the characteristic flattop in their electrostatic potential profile becomes noticeable only when $M_{d l}-M$ or $\left|M-M_{t r}\right|$ are very small, of order $10^{-5}$ or lower. The onset of their flat-top character is more clearly seen in the corresponding electric field or charge density profiles.

3. However, even when $M_{d l}-M$ or $\left|M-M_{t r}\right|$ is tiny (of order $10^{-16}$ for the double layers), at the limit of the numerical accuracy, their potential profiles are still soliton-like, with the same boundary conditions left and right, unlike the asymmetric forms of double layers or triple root structures.

4. So although the Sagdeev pseudopotential changes continuously when $M \rightarrow M_{d l}$ or $M \rightarrow M_{t r}$, the character of the profiles changes discontinuously at the velocity of the double layer or triple root structure itself. This part of our conclusions was not quite expected.

5. For a sufficiently wide flattop, seen as such on the graphs, the electric field signature looks like two unipolar signals with opposite polarities, separated by the length of the flat-top part of the electrostatic potential. Unipolar electric field profiles are the signature of both double layers and triple root structures. However, there seems to be little physical or mathematical reason to identify the electric field signature of a flat-top soliton with either a pair of opposite polarity double layers or an amalgamation of a double layer and a soliton.

6. Nevertheless, their extremely limited existence range renders observations of flat-top solitons unlikely, unless helped by a fortunate stroke of serendipity. This remark should be qualified because double layers have been observed, despite the fact that they exist for only one value of $M$. This implies that the range of Mach numbers alone is not an indication of the likelihood of existence, even if the range has a zero measure or is very narrow. Seemingly, nature produces double layers, which might suggest that the precision in velocity at which the double layer is supposed to exist can cover a range in initial conditions. Alternatively, it may be that double layers are particularly robust structures, and due to their stability, these are observable despite their narrow existence range. If that is the case, one might conjecture that one is unlikely to observe flat-top structures at all, with speeds that are theoretically extremely close to the double layer velocity.

7. This is one of several aspects of the properties of flat-top solitons that need to be investigated by numerical simulation, including stability and possible interactions.

All in all, what we can definitely say about flat-top solitons is that their theoretical existence requires the system to satisfy very stringent conditions, that these may be difficult to achieve (as is theoretically the case for double layers and a fortiori for triple root structures), that we are not aware of any observations, and, unfortunately, that the final transition from the flat-top to double layer shape seems to be discontinuous. This is a topic where suitable numerical simulations are required, with a focus on generation mechanisms of flat-top solitons, their stability, and their robustness with respect to collisions with other solitons.

\section{ACKNOWLEDGMENTS}

We thank Dr. J. S. Pickett (University of Iowa) for enlightening discussions.

\section{APPENDIX A: GENERIC CHARACTER OF FLAT-TOP STRUCTURES}

Consider solitons in the velocity range $M_{s}<M<M_{d l}$, where $M_{s}$ is an acoustic speed and $M_{d l}$ is the double layer velocity. Let $\varphi_{r}(M)$ be the root of the Sagdeev pseudopotential (also acting as the soliton amplitude). We assume that the following limit exists:

$$
\lim _{M \rightarrow M_{d l}} \frac{\partial S}{\partial \varphi}\left(\varphi_{r}, M\right)=0 .
$$

By setting $\varphi=\varphi_{r}+\delta \varphi$, a Taylor series expansion of the Sagdeev pseudopotential about $\varphi=\varphi_{r}$ yields $^{26}$

$$
S\left(\varphi_{r}+\delta \varphi, M\right)=S\left(\varphi_{r}, M\right)+\frac{\partial S}{\partial \varphi}\left(\varphi_{r}, M\right) \delta \varphi+\mathcal{O}\left(\delta \varphi^{2}\right) .
$$

Here, the first term on the right-hand side vanishes as $\varphi_{r}$ is a root. For $|\delta \varphi| \ll 1$, we may, therefore, approximate the Sagdeev pseudopotential about $\varphi_{r}$ as follows:

$$
S\left(\varphi_{r}+\delta \varphi, M\right) \simeq \frac{\partial S}{\partial \varphi}\left(\varphi_{r}, M\right) \delta \varphi
$$

By substituting the approximation (A3) into the energy-like equation, 


$$
\frac{1}{2}\left(\frac{d \varphi}{d \xi}\right)^{2}+S(\varphi, M)=0,
$$

one obtains the following separable equation:

$$
\frac{d \varphi}{d \xi} \simeq \pm \sqrt{-2 \frac{\partial S}{\partial \varphi}\left(\varphi_{r}, M\right) \delta \varphi .}
$$

This equation can be readily integrated to obtain the following general solution:

$$
\varphi(\xi) \simeq \varphi_{r}-\frac{1}{2} \frac{\partial S}{\partial \varphi}\left(\varphi_{r}, M\right)\left(\xi-\xi_{0}\right)^{2},
$$

where $\xi_{0}$ is a constant of integration. In order to center the soliton at $\xi=0$, we set $\xi_{0}=0$ to get the solution,

$$
\varphi(\xi) \simeq \varphi_{r}-\frac{1}{2} \frac{\partial S}{\partial \varphi}\left(\varphi_{r}, M\right) \xi^{2} .
$$

It should be emphasized that this is a local solution only in the sense that it is only valid for $|\delta \varphi| \ll 1$.

To approximate the width of the flattop, we calculate the $\xi$ length between the amplitude $(\xi=0)$ and the value $\xi^{*}$ where the potential is $99 \%$ of the amplitude, i.e., where

$$
\xi^{*} \text { satisfies } \varphi\left(\xi^{*}\right)=0.99 \varphi_{r} \text {. }
$$

Substituting $\xi^{*}$ into the approximate solution (A7) gives

$$
\xi^{*} \simeq \pm \sqrt{\frac{\varphi_{r}}{50 \frac{\partial S}{\partial \varphi}\left(\varphi_{r}, M\right)}} .
$$

By choosing the + sign, we choose the point to the right of $\xi=0$ where $\varphi=0.99 \varphi_{r}$, given by

$$
\xi^{*} \simeq \sqrt{\frac{\varphi_{r}}{50 \frac{\partial S}{\partial \varphi}\left(\varphi_{r}, M\right)}} .
$$

We now consider the limit when $M \rightarrow M_{d l}$. Since $\frac{\partial S}{\partial \varphi}\left(\varphi_{r}, M\right)$ $\rightarrow 0$, it follows that $\xi^{*} \rightarrow+\infty$. In other words, the width of the flat-top part of the solution is unbounded, corresponding to the existence of flat top solutions with arbitrarily large width.

This proof holds for flat-top solitons near both positive and negative double layers, i.e., both $\varphi_{r}>0$ and $<0$. Remarkably, it can also be used for flat-tops near both positive and negative triple root structures, applying to $M<M_{t r}$ as well as to $M>M_{t r}$.

Trying to give an analogous proof based on a Taylor expansion of $\varphi(\xi)$ is not helpful. Indeed, $S$ is a function of $\varphi$, and, in turn, $\varphi$ is a function of $\xi$, so that may be regarded as being a roundabout procedure. Moreover, importantly, the range of applications of such a Taylor expansion is limited to $|\xi|<1$, but as seen in the figures, the flat top part of the soliton profiles spans a much wider range in $\xi$.

\section{APPENDIX B: MODEL PLASMA WITH DOUBLE LAYERS}

This dusty plasma composition ${ }^{16}$ contains three species: hot electrons, nonthermal positive ions, and cold, negatively charged dust grains, with respective subscripts $e$, $i$, and $d$. Following a Cairns nonthermal distribution, ${ }^{17}$ the normalized positive ion density $n_{i}$ is given at the macroscopic level by

$$
n_{i}=\left(1+\beta \varphi+\beta \varphi^{2}\right) \exp [-\varphi],
$$

where $\beta$ is the nonthermality parameter. In common with many descriptions of dusty plasma waves, the very mobile electrons have been assumed to be Boltzmann-distributed, in normalized form,

$$
n_{e}=(1-f) \exp [\tau \varphi],
$$

where $\tau=T_{i} / T_{e}$ is the ion-to-electron temperature ratio. From the combination of the cold dust continuity and momentum equations, written in a frame where the solitary structure is stationary, the dust density is obtained, after integration with respect to $\xi$, the comoving spatial coordinate,

$$
n_{d}=\frac{f}{\sqrt{1+\frac{2 \varphi}{M^{2}}}} .
$$

Here, $f$ is the fractional negative charge on the dust species. The basic set of equations is closed by Poisson's equation,

$$
\frac{d^{2} \varphi}{d \xi^{2}}=n_{e}+n_{d}-n_{i},
$$

which after integration leads to the Sagdeev energy integral

$$
\frac{1}{2}\left(\frac{d \varphi}{d \xi}\right)^{2}+S(\varphi, M)=0 .
$$

In this, the Sagdeev pseudopotential $S(\varphi, M)$ plays the role of a potential energy, in suitably normalized variables. The expression of $S(\varphi, M)$ has been given in Sec. II, in (1), and further details can be taken from the original paper. ${ }^{16}$

\section{APPENDIX C: MODEL PLASMA WITH TRIPLE ROOT STRUCTURES}

The model includes nonthermal electrons and singly charged ions, and cold negative and positive dust grains. ${ }^{15}$ The fraction of charge residing on the negative dust is here $f=Z_{d n} n_{d n 0} / n_{0}$ and on the electrons $1-f=n_{e 0} / n_{0}$, whereas the fraction of charge residing on the positive dust is $g=Z_{d p} n_{d p 0} / n_{0}$ and on the ions $1-g=n_{i 0} / n_{0}$. Here, $n_{0}=n_{e 0}+Z_{d n} n_{d n 0}=n_{i 0}+Z_{d p} n_{d p 0}$, where $Z$ refers to the absolute value of the dust charges and the other symbols have their usual meaning.

The normalized electron and ion densities are given for Boltzmann distributions as

$$
\begin{gathered}
n_{e}=(1-f) \exp [\varphi], \\
n_{i}=(1-g) \exp [-\varphi] .
\end{gathered}
$$

These are culled from the original paper, ${ }^{15}$ but reduced to the special case of Boltzmann rather than Cairns nonthermal distributions. The cold dust is described by the continuity and momentum equations in normalized variables, from which the normalized dust densities follow as: 


$$
\begin{gathered}
n_{d n}=\frac{f}{\sqrt{1+\frac{2 \varphi}{M^{2}}}}, \\
n_{d p}=\frac{g}{\sqrt{1-\frac{2 \varphi}{\mu M^{2}}}} .
\end{gathered}
$$

Here, $\mu=m_{d p} Z_{d n} / Z_{d p} m_{d n}$ is the ratio of the mass-to-charge ratios of the two dust species. Further details can be found in the original paper, ${ }^{15}$ where the normalization is also discussed. Altogether, this leads again to (B5), with $S(\varphi, M)$ given in (3).

\section{DATA AVAILABILITY}

Data sharing is not applicable to this article as no new data were created or analyzed in this study.

\section{REFERENCES}

${ }^{1}$ R. Z. Sagdeev, "Cooperative phenomena and shock waves in collisionless plasmas," Reviews of Plasma Physics, edited by M. A. Leontovich (Consultants Bureau, New York, 1960), Vol. 4, pp. 23-91.

${ }^{2}$ A. E. Dubinov and D. Yu. Kolotkov, IEEE Trans. Plasma Sci. 40, 1429-1433 (2012).

${ }^{3}$ A. E. Dubinov and D. Y. Kolotkov, Plasma Phys. Rep. 38, 909-912 (2012).

${ }^{4}$ A. Das, A. Bandyopadhyay, and K. P. Das, J. Plasma Phys. 78, 149-164 (2012).

${ }^{5}$ F. Verheest, M. A. Hellberg, and I. Kourakis, Phys. Plasmas 20, 012302 (2013).

${ }^{6}$ F. Verheest, M. A. Hellberg, and I. Kourakis, Phys. Rev. E 87, 043107 (2013).

${ }^{7}$ F. Verheest, M. A. Hellberg, and I. Kourakis, Phys. Plasmas 20, 082309 (2013).

${ }^{8}$ M. A. Hellberg, T. K. Baluku, F. Verheest, and I. Kourakis, J. Plasma Phys. 79, 1039-1043 (2013).

${ }^{9}$ F. Verheest, G. S. Lakhina, and M. A. Hellberg, Phys. Plasmas 21, 062303 (2014).

${ }^{10}$ F. Verheest and M. A. Hellberg, Phys. Plasmas 22, 012301 (2015).

${ }^{11}$ S. V. Singh and G. S. Lakhina, Commun. Nonlinear Sci. Numer. Simul. 23, 274-281 (2015).

${ }^{12}$ S. V. Steffy and S. S. Ghosh, Phys. Plasmas 25, 062302 (2018).

${ }^{13}$ P. G. Drazin and R. S. Johnson, Solitons: An Introduction (Cambridge University Press, UK, 1989).

${ }^{14}$ F. Verheest, Phys. Plasmas 17, 062302 (2010).

${ }^{15}$ F. Verheest and C. P. Olivier, Phys. Plasmas 24, 113708 (2017).

${ }^{16}$ F. Verheest and S. R. Pillay, Phys. Plasmas 15, 013703 (2008).
${ }^{17}$ R. A. Cairns, A. A. Mamun, R. Bingham, R. Boström, R. O. Dendy, C. M. C. Nairn, and P. K. Shukla, Geophys. Res. Lett. 22, 2709-2712, https://doi.org/ 10.1029/95GL02781 (1995).

${ }^{18}$ F. Verheest, M. A. Hellberg, and I. Kourakis, Phys. Plasmas 15, 112309 (2008).

${ }^{19}$ F. Verheest, Phys. Plasmas 16, 013704 (2009).

${ }^{20}$ F. Verheest and M. A. Hellberg, Phys. Plasmas 17, 102312 (2010).

${ }^{21}$ F. Verheest, Phys. Plasmas 18, 083701 (2011).

${ }^{22}$ F. Verheest, M. A. Hellberg, and T. K. Baluku, Phys. Plasmas 19, 032305 (2012).

${ }^{23}$ F. Verheest and M. A. Hellberg, Phys. Plasmas 24, 022306 (2017).

${ }^{24}$ F. Verheest, J. Phys. A 42, 285501 (2009).

${ }^{25}$ S. Sundar, A. Das, V. Saxena, P. Kaw, and A. Sen, Phys. Plasmas 18, 112112 (2011).

${ }^{26}$ C. P. Olivier, F. Verheest, and S. K. Maharaj, J. Plasma Phys. 83, 905830605 (2017).

${ }^{27}$ R. E. Ergun, C. W. Carlson, J. P. McFadden, F. S. Mozer, G. T. Delory, W. Peria, C. C. Chaston, M. Temerin, R. Elphic, R. Strangeway, R. Pfaff, C. A. Cattell, D. Klumpar, E. Shelley, W. Peterson, E. Moebius, and L. Kistler, Geophys. Res. Lett. 25, 2025-2028, https://doi.org/10.1029/98GL00635 (1998).

${ }^{28}$ R. E. Ergun, C. W. Carlson, J. P. McFadden, F. S. Mozer, G. T. Delory, W. Peria, C. C. Chaston, M. Temerin, I. Roth, L. Muschietti, R. Elphic, R. Strangeway, R. Pfaff, C. A. Cattell, D. Klumpar, E. Shelley, W. Peterson, E. Moebius, and L. Kistler, Geophys. Res. Lett. 25, 2041-2044, https://doi.org/ 10.1029/98GL00636 (1998).

${ }^{29}$ J. P. McFadden, C. W. Carlson, R. E. Ergun, F. S. Mozer, L. Muschietti, I. Roth, and E. Moebius, J. Geophys. Res. 108, 8018, https://doi.org/10.1029/ 2002JA009485 (2003).

${ }^{30}$ M. Temerin, K. Cerny, W. Lotko, and F. S. Mozer, Phys. Rev. Lett. 48, 1175-1178 (1982).

${ }^{31}$ R. E. Ergun, Y.-J. Su, L. Andersson, C. W. Carlson, J. P. McFadden, F. S. Mozer, D. L. Newman, M. V. Goldman, and R. J. Strangeway, Phys. Rev. Lett. 87, 045003 (2001).

${ }^{32}$ R. E. Ergun, L. Andersson, J. Tao, V. Angelopoulos, J. Bonnell, J. P. McFadden, D. E. Larson, S. Eriksson, T. Johansson, C. M. Cully, D. N. Newman, M. V. Goldman, A. Roux, O. LeContel, K.-H. Glassmeier, and W. Baumjohann, Phys. Rev. Lett. 102, 155002 (2009).

${ }^{33}$ J. S. Pickett, L.-J. Chen, R. L. Mutel, I. W. Christopher, O. Santolík, G. S. Lakhina, S. V. Singh, R. V. Reddy, D. A. Gurnett, B. T. Tsurutani, E. Lucek, and B. Lavraud, Adv. Space Res. 41, 1666-1676 (2008).

${ }^{34}$ F. S. Mozer, O. A. Agapitov, A. Artemyev, J. L. Burch, R. E. Ergun, B. L. Giles, D. Mourenas, R. B. Torbert, T. D. Phan, and I. Vasko, Phys. Rev. Lett. 116, 145101 (2016). 